Meta

Journal des traducteurs

Translators' Journal

\title{
Le master européen de traduction : un projet de la direction générale de la traduction de la Commission européenne
}

\section{Angeliki Petrits}

Volume 52, numéro 1, mars 2007

Traductologie : une science cognitive

URI : https://id.erudit.org/iderudit/014724ar

DOI : https://doi.org/10.7202/014724ar

Aller au sommaire du numéro

Éditeur(s)

Les Presses de l'Université de Montréal

ISSN

0026-0452 (imprimé)

1492-1421 (numérique)

Découvrir la revue

Citer cet article

Petrits, A. (2007). Le master européen de traduction : un projet de la direction générale de la traduction de la Commission européenne. Meta, 52(1), 85-92. https://doi.org/10.7202/014724ar
Résumé de l'article

De nombreux pays européens possèdent une longue tradition de formation au métier de traducteur. Dans d'autres en revanche, ces formations n'existent pas encore ou n'ont été que récemment mises sur pied. La Commission européenne, qui emploie un nombre considérable de traducteurs dans 20 langues officielles, est un acteur majeur du marché européen de la traduction. Elle a entrepris plusieurs actions en vue de créer un rapprochement avec le monde universitaire. Le projet d'un master européen de traduction présenté ici vise, parallèlement à d'autres actions, à harmoniser les programmes universitaires de traduction et ainsi, à garantir une formation de qualité pour les traducteurs à travers l’Union européenne. 


\title{
Le master européen de traduction: un projet de la direction générale de la traduction de la Commission européenne
}

\author{
ANGELIKI PETRITS \\ Direction générale de la traduction \\ Commission européenne \\ angelique.petrits@ec.europa.eu
}

\begin{abstract}
RÉSUMÉ
De nombreux pays européens possèdent une longue tradition de formation au métier de traducteur. Dans d'autres en revanche, ces formations n'existent pas encore ou n'ont été que récemment mises sur pied. La Commission européenne, qui emploie un nombre considérable de traducteurs dans 20 langues officielles, est un acteur majeur du marché européen de la traduction. Elle a entrepris plusieurs actions en vue de créer un rapprochement avec le monde universitaire. Le projet d'un master européen de traduction présenté ici vise, parallèlement à d'autres actions, à harmoniser les programmes universitaires de traduction et ainsi, à garantir une formation de qualité pour les traducteurs à travers l'Union européenne.
\end{abstract}

\begin{abstract}
While many European countries have a long tradition of translation training, some others still lack such programmes or have begun to develop them only recently. The European Commission, which is a major employer of translators in 20 official languages, is an important player on the European translation market. It has taken several actions in order to establish closer links with universities. The project of a European Masters in Translation that we present in this paper aims, together with the other actions, at harmonising translation programmes in order to guarantee quality training for translators within the EU.
\end{abstract}

\section{MOTS-CLÉS/KEYWORDS}

commission europénne, direction générale de la traduction, master européen de traduction, formation des traducteurs, traducteur invité

\section{Introduction}

La direction générale de la traduction (DGT) de la Commission européenne, le plus grand service de traduction au monde, compte environ 2000 traducteurs qui, partagés entre les sièges de Bruxelles et de Luxembourg, assurent de manière rigoureuse la communication écrite de la Commission. Au cours de la seule année 2005, la DGT a traduit plus d'un million trois cent mille pages en 20 langues officielles.

Même si les vingt langues officielles actuelles ont le même statut au sein des institutions européennes, la Commission, pour des raisons d'efficacité, a adopté trois langues de travail internes, à savoir le français, l'anglais et l'allemand (langues procédurales). Les documents destinés aux réunions de la Commission sont ainsi présentés dans ces trois langues de travail, mais seule leur version définitive ultérieurement transmise aux autres institutions sera traduite dans toutes les langues officielles. 
En 2005, le nombre des originaux rédigés en anglais a été de $68 \%$, ce qui représente une augmentation de $6 \%$ par rapport à 2004; les originaux français n'ont atteint que $16 \%$ du volume total, soit une diminution de $10 \%$ par rapport à 2004; quant à l'allemand, il a enregistré une légère hausse avec près de $4 \%$ des originaux; l'ensemble des autres langues a représenté un peu plus de $11 \%$ du volume total.

Le $1^{\text {er }}$ mai 2004, un élargissement sans précédent a eu lieu: l'Union européenne (UE) a accueilli dix nouveaux États membres et neuf langues officielles se sont ajoutées aux onze langues existantes, portant ainsi leur nombre à vingt. Le $1^{\text {er }}$ janvier 2007, l'irlandais deviendra la $21^{\mathrm{e}}$ langue officielle de l'UE.

\section{Les répercussions de l'élargissement sur le marché de la traduction}

Du fait de l'augmentation du nombre de langues officielles, la demande de traduction s'est accrue d'un tiers. Bien que l'accroissement de la demande par langue officielle ait été le même que lors des élargissements précédents, les départements de traduction des neuf nouvelles langues n'avaient pas encore atteint leur pleine capacité en nombre de traducteurs le $1^{\text {er }}$ mai 2004.

Pour pallier le manque de traducteurs dans ces langues, la Commission, d'une part, a adopté une stratégie visant à aligner l'offre et la demande de traduction; plus concrètement, elle a décidé de limiter la longueur des documents à traduire et d'accorder la priorité à la traduction des textes législatifs et des documents politiques importants. D'autre part, elle a eu massivement recours à la traduction externe en attendant d'avoir pu recruter suffisamment de traducteurs des nouvelles langues.

Devant les difficultés de recrutement rencontrées, la nécessité de créer une filière universitaire pour former des traducteurs capables de travailler au service des institutions européennes a commencé à se faire sentir. C'est dans ce contexte qu'est né le projet du master européen de traduction (EMT - European Masters in Translation). Le projet a en outre vu le jour au moment même où les établissements universitaires étaient en train d'adapter leurs programmes au processus de Bologne.

\section{Assistance pédagogique}

Alors que la plupart des anciens États membres de l'UE avaient une longue tradition dans le domaine de la formation des traducteurs, cette formation était inadaptée, voire inexistante, dans certains des nouveaux États.

Dans un premier temps, la DGT a décidé de fournir assistance et soutien aux nouveaux États en vue d'identifier leurs besoins en matière de formation des traducteurs. Dans un deuxième temps, elle a proposé de les aider à satisfaire ces besoins.

À cet effet, elle a développé un programme de cours <http://ec.europa.eu/dgs/ translation/external_relations/universities/master_curriculum_en.pdf $>$ pour un master européen de traduction. Les universités de l'UE sont invitées à adapter leurs propres programmes selon ce programme qui se veut minimaliste. Les matières proposées (voir annexe) sont celles qui doivent faire l'objet d'un enseignement obligatoire, mais d'autres matières peuvent être ajoutées en fonction des particularités du pays et des besoins du marché local.

Le programme proposé vise à la fois à former des traducteurs de haut niveau possédant toutes les qualifications requises pour exercer la profession et à procurer 
aux institutions de l'UE un nombre suffisant de traducteurs qui puissent répondre à leurs besoins spécifiques de traduction.

\section{Type de formation}

À l'instar de l'EMCI (European Masters in Conference Interpreting), l'EMT est conçu comme un programme de formation intensive à la traduction s'étalant sur deux ans maximum et comportant de 60 à 100 crédits (d'après le système européen de transfert de crédits).

La formation doit être axée sur la composante «traduction» et non sur l'apprentissage des langues dont la connaissance est une condition préalable.

\section{Profil du traducteur}

Le programme a été développé selon le profil du traducteur de la Commission européenne $<$ http://ec.europa.eu/dgs/translation/workingwithus/recruitment/translator_ profile_fr.htm $>$.

Selon ce profil, le traducteur type doit avoir une connaissance parfaite de tous les aspects de la langue cible (la langue principale) ainsi qu'une maîtrise approfondie de deux autres langues officielles, de préférence parmi les langues procédurales de la Commission (le français, l'anglais ou l'allemand). En même temps, il doit posséder des connaissances thématiques dans un large spectre de registres et de sujets ainsi qu'une connaissance du contexte culturel des pays dans lesquels ces langues sont parlées.

Les aptitudes intellectuelles requises ne sont pas moins importantes: la capacité d'appréhender les problèmes de toute nature, souvent complexes, d'effectuer une recherche terminologique approfondie, d'utiliser à bonne fin les outils informatiques ainsi que de communiquer efficacement font partie des qualifications requises par les traducteurs des institutions européennes.

\section{Critères d'admission}

Les candidats au master européen de traduction doivent être en possession d'un diplôme universitaire correspondant à un cycle complet de trois années au moins. Ils doivent prouver leur connaissance parfaite de la langue principale ainsi que la connaissance approfondie de deux langues étrangères lors d'un examen d'admission. Cet examen doit également permettre d'évaluer leur culture générale ainsi que leurs aptitudes intellectuelles au métier de traducteur (voir ci-dessus).

Il est important que les lauréats de toute discipline soient admis à l'examen et non pas uniquement les lauréats des facultés de lettres ou de sciences humaines. La Commission recrute des universitaires de tous les domaines qui seront appelés à traduire des textes très spécialisés allant de la médecine au droit en passant par l'ingénierie ou les finances. La connaissance approfondie de ces domaines constitue une garantie pour la qualité de la traduction.

\section{Collaboration avec les universités}

La DGT a entrepris plusieurs actions de collaboration avec les universités. 


\section{a. Assistance}

En plus du programme de l'EMT qu'elle a élaboré et qu'elle souhaite faire adopter par les universités qui le désirent, la DGT envoie ses représentants dans les établissements qui souhaitent adapter leurs programmes en vue de les aider à concrétiser ce projet.

\section{b. Traducteurs invités (VTS)}

Elle a institué le programme de traducteur invité <http://ec.europa.eu/dgs/translation/external_relations/universities/visiting_translator_fr.htm $>$ selon lequel des traducteurs expérimentés de la DGT séjournent de quatre à six semaines dans une université de l'UE. Ils enseignent la traduction mais en plus donnent des conférences sur plusieurs aspects du travail à la DGT ainsi que sur la politique du multilinguisme à la Commission. Les questions traitées incluent les méthodes de travail, la vie d'un document législatif de sa naissance à sa traduction, les outils d'aide à la traduction et la politique de recrutement.

La DGT libère le traducteur de ses tâches habituelles pendant la période de visite et prend en charge les frais de voyage et de séjour. En compensation, l'université qui accueille le traducteur doit offrir des cours de langue à ce dernier pour qu'il puisse améliorer ses connaissances de la langue du pays.

\section{c. Séjours de formation}

Selon les disponibilités du service et en entente avec les universités, des étudiants de dernière année sont acceptés de manière sélective pour un séjour de formation non rémunéré de quatre semaines. Ils doivent être capables de traduire à partir de deux langues officielles vers une troisième langue officielle (langue principale) et, en principe, ils intégreront pendant la période de leur séjour le département linguistique de cette langue. Cette action leur permettra d'élargir leurs connaissances aux méthodes de travail de la DGT et peut s'avérer une expérience utile pour l'avenir.

Sur demande des universités, la DGT organise également des visites de un ou deux jours proposant un programme sur mesure aux groupes d'étudiants et d'enseignants qui veulent se familiariser avec les méthodes de travail de la DGT.

\section{d. Stages administratifs}

Comme tous les services de la Commission, la DGT accueille deux fois par an des stagiaires rémunérés pour une période de cinq mois <http://ec.europa.eu/stages/ information/application_fr.htm $>$. Les critères de recevabilité incluent la formation de niveau universitaire ainsi que la capacité de traduire à partir de deux langues officielles (dont une doit être le français, l'anglais et l'allemand) vers une troisième langue officielle. Toutes les nationalités sont admises et la sélection se fait à deux niveaux: dans un premier temps centralement par la Commission et par la suite par la DGT selon les langues et les profils requis. 


\section{e. Conférence EMT 2006}

Le 19 et 20 octobre 2006, la DGT organisera sa première conférence avec les universités. Elle s'inscrit clairement dans le cadre de la politique sur le multilinguisme de la Commission telle qu'elle est exprimée dans la communication du 22 novembre 2005.

Le titre Vers un master européen de traduction se veut dynamique et marque un processus vers un objectif précis. Des experts de tous les États membres seront invités à Bruxelles pour débattre des questions relatives à la formation des traducteurs dans l'UE et des moyens de mettre en place un master européen de traduction.

Autres objectifs de cette conférence:

- Créer un rapprochement entre les universités où la traduction est enseignée et la Commission, un des plus importants employeurs de traducteurs à l'échelle mondiale.

- Promouvoir le programme de l'EMT auprès des universités de l'UE et des pays en voie d'adhésion.

- Créer un réseau d'universités à travers l'UE prêtes à collaborer dans le cadre de l'EMT.

- Encourager les universités à adapter leurs programmes selon le programme proposé par la DGT.

\section{Conclusion}

Les différentes actions de rapprochement avec les universités entreprises par la DGT visent à promouvoir le métier du traducteur en tant que profession à part entière. Nous espérons qu'elles contribueront à obtenir sur le plan européen un enseignement de la traduction de qualité, capable de fournir des professionnels de haut niveau tant aux institutions européennes qu'au marché en général.

\section{ANNEXE}

Master de traduction

Description détaillée de la composition du programme d'études proposée

Direction générale de la traduction, Commission européenne

\section{La traduction en tant que profession}

Objectif

Familiariser les étudiants avec différents aspects du métier de traducteur pour les aider à développer un sentiment marqué d'appartenance à la profession et à décider de l'orientation de leur carrière.

\section{Contenu indicatif}

L'histoire de la traduction et de la profession de traducteur, l'évolution et les tendances de la profession, l'éthique et les bonnes pratiques professionnelles, les organisations et réseaux de traducteurs, les questions de droits d'auteur, les perspectives de carrière dans le secteur de la traduction, la rémunération, le traducteur en tant que chef d'entreprise, etc.

\section{Observations}

Il est souhaitable que des traducteurs professionnels expérimentés participent à cette partie du programme. À titre d'exemple, ce volet pourrait comporter une série de présentations sur différents domaines et aspects de la traduction effectuées par des traducteurs invités. 


\section{La théorie de la traduction}

\section{Objectif}

Fournir de solides bases théoriques à l'exercice de la profession et faciliter une compréhension exhaustive des questions fondamentales sous-tendant le travail concret du traducteur.

\section{Observations}

Les grandes questions et principaux «sujets de controverse» de la théorie de la traduction, les approches et concepts essentiels ainsi que les principales évolutions de la pensée dans ce domaine, la littérature spécialisée et les publications fondamentales sur ce sujet.

\section{L'analyse du texte/discours et l'analyse des demandes de traduction}

\section{Objectif}

Fournir aux étudiants les moyens conceptuels permettant d'analyser les textes à traduire ainsi que les différents aspects de la demande et de la situation même de traduction afin qu'ils puissent élaborer des textes adaptés à leur finalité. Leur donner les moyens de repérer et décrire d'éventuels problèmes linguistiques, textuels et stratégiques et de justifier leurs choix sur chacun de ces aspects.

\section{Contenu indicatif}

Concepts fondamentaux de la linguistique et de l'analyse du texte/discours; principales caractéristiques de la textualité; structures thématiques, fonctionnelles et argumentatives des textes; caractéristiques des types de texte; analyse des situations et demandes de traduction et des fonctions de la traduction.

\section{Observations}

L'analyse du texte/discours devrait être orientée sur les besoins du traducteur.

\section{La communication interculturelle}

\section{Objectif}

Sensibiliser les étudiants aux différences culturelles, au rôle des éléments culturels dans la traduction et, plus généralement, dans la communication internationale; leur fournir les outils conceptuels les aidant à interpréter ces données culturelles.

\section{Contenu indicatif}

La relation entre langue et culture; les spécificités culturelles et leurs manifestations textuelles; le rôle du traducteur en tant que "médiateur culturel», les problèmes de traduction résultant des différences de culture, les méthodes contrastives (telle que la rhétorique contrastive), les traductions en tant que sources d'influence culturelle.

\section{Le travail terminologique}

\section{Objectif}

Introduire les principes fondamentaux du travail terminologique et permettre aux étudiants d'acquérir une connaissance pratique et une maîtrise suffisante de la gestion de la terminologie et de l'utilisation des outils terminologiques.

\section{Contenu indicatif}

Les principes fondamentaux du travail terminologique, les banques de termes et autres ressources terminologiques, les outils informatiques de terminologie, la gestion de la terminologie et les principes de base de la lexicographie. 


\section{Les technologies de l'information au service de la traduction}

\section{Objectif}

Permettre aux étudiants d'acquérir une connaissance pratique et une maîtrise suffisante du traitement électronique des documents, de l'utilisation des réseaux d'information et des outils et technologies d'aide à la traduction.

\section{Contenu indicatif}

Le traitement et la gestion électroniques des documents, les systèmes de gestion des traductions, les réseaux, Internet en tant que source d'information, les outils de recherche, les bases de données électroniques, la traduction assistée par ordinateur (dictionnaires en ligne, correcteurs orthographiques et grammaticaux, mémoires de traduction et outils informatisés de traduction tels que les systèmes de reconnaissance vocale), la traduction automatique.

\section{La sensibilité linguistique et le développement des compétences langagières}

\section{Objectif}

Sensibiliser les étudiants à l'exactitude linguistique, stylistique et textuelle ainsi qu'à la nécessité d'une formulation «naturelle», notamment dans leur première langue et du point de vue de la communication transnationale.

\section{Contenu indicatif}

Les principes de base de la préservation et de l'enrichissement du bagage linguistique, l'évolution actuelle de la (première) langue, les traductions en tant que sources d'influence linguistique, les interférences linguistiques et la traduction, la révision et le contrôle orthographique et grammatical des traductions.

\section{La connaissance de domaines spécialisés et de leur langue}

\section{Objectif}

Assurer une compréhension minimale de certains domaines spécialisés - de leurs principaux concepts et de leurs conventions linguistiques (différentes d'une culture à l'autre) et pratiques (types de texte) - afin d'étoffer les compétences thématiques et la capacité de communication des étudiants.

\section{Contenu indicatif}

Une introduction à différents domaines particuliers, à leur cadre conceptuel, à leur langue de spécialité et à leur terminologie. Citons comme domaines possibles le droit et l'administration, l'économie et le commerce, les sciences et la recherche, la technologie, la médecine et les organisations internationales.

\section{Observations}

Avec les cours de "pratique de la traduction", cette partie devrait constituer une composante majeure de la formation, les deux devant représenter au moins $50 \%$ du nombre total d'unités de cours.

Compte tenu de l'étendue des domaines spécialisés, seul un nombre limité d'entre eux ( 1 ou 2) devrait être ciblé. À cet égard, il serait intéressant de tirer parti des éventuelles études accomplies antérieurement par les étudiants dans des domaines spécialisés. Une formation sur les organisations internationales, notamment l'UE et ses institutions, serait utile aux futurs traducteurs de l'Union européenne.

\section{La pratique de la traduction (concernant plus d'une combinaison linguistique)}

\section{Objectif}

Réunir et affiner les connaissances et compétences acquises grâce à des exercices guidés de traduction pour permettre aux étudiants de devenir des traducteurs professionnels, capables de fournir dans la langue cible des textes adaptés à leur fonction et acceptables sur le marché. 


\section{Contenu indicatif}

Divers types de demandes de traduction répondant à des fonctions différentes, reflétant des catégories de texte et des domaines disparates et nécessitant un traitement distinct des textes sources (traduction soumise à de strictes contraintes d'équivalence, traduction interprétative, rédaction de résumés, etc.). La révision, l'analyse et la critique de traductions, le travail terminologique lié aux textes traduits.

\section{Observations}

Avec les cours sur la «connaissance des domaines spécialisés et de leur langue», cette partie devrait constituer une composante majeure de la formation, les deux devant représenter au moins $50 \% \mathrm{du}$ nombre total d'unités de cours.

L'idéal serait qu'il existe plusieurs modules de traduction, dont au moins un (le premier) se limiterait à des textes de nature générale, tandis que les autres pourraient/devraient proposer des textes dans des domaines plus pointus (de préférence ceux abordés dans le volet sur la connaissance des domaines spécialisés et de leur langue). Un cours de traduction sur la thématique européenne serait utile aux futurs traducteurs de l'UE.

Toutes les traductions demandées devraient être réalistes, autrement dit porter sur des textes qui sont ou pourraient être traduits dans la réalité, pour lesquels une véritable demande de traduction et la fonction du texte cible peuvent être définies. Certains exercices de traduction pourraient inclure une partie explicative contenant les observations de l'étudiant sur son propre travail. Les étudiants devraient aussi réviser et commenter les traductions de leurs homologues ou de traducteurs. Pour l'enseignement de la traduction et l'évaluation des travaux des étudiants, il conviendrait au besoin de faire appel à des traducteurs professionnels expérimentés.

Dans la perspective d'un éventuel futur emploi au sein de l'UE, des cours de traduction dans la première langue à partir d'au moins deux langues communautaires seraient souhaitables. De même, des cours de traduction de la première langue vers au moins une langue (étrangère) seraient utiles. Pour ces traductions de la langue A vers la langue B ou C, l'objectif de la formation est de développer l'aptitude à fournir des traductions pouvant facilement faire l'objet d'un contrôle/d'une révision d'une ampleur raisonnable par un locuteur natif et aboutissant, dans la langue cible, à des textes adaptés à leur fonction et acceptables sur le marché.

Pour effectuer leurs traductions et tous leurs autres travaux écrits, les étudiants devraient recourir de manière active aux outils électroniques mentionnés ci-dessus dans les rubriques sur les «technologies de l'information au service de la traduction» et le «travail terminologique». 